\title{
Efficient Enzymatic Production of the Bacterial Second Messenger c-di-GMP by the Diguanylate Cyclase YdeH from $E$. coli
}

\author{
Franziska Zähringer • Claudia Massa • \\ Tilman Schirmer
}

Received: 19 January 2010 / Accepted: 15 June 2010 /

Published online: 29 June 2010

(C) Springer Science+Business Media, LLC 2010

\begin{abstract}
Cyclic di-GMP (c-di-GMP) is an almost universal bacterial second messenger involved in the regulation of cell surface-associated traits and the persistence of infections. GGDEF and EAL domain-containing proteins catalyse c-di-GMP synthesis and degradation, respectively. We report the enzymatic large-scale synthesis of c-di-GMP, making use of the GGDEF domain-containing protein YdeH from Escherichia coli. Overexpression and purification of YdeH have been established, and the conditions for c-di-GMP synthesis were optimised. In contrast to the chemical synthesis of c-di-GMP, enzymatic c-di-GMP production is a one-step reaction that can easily be performed with the equipment of a standard biochemical lab. The protocol allows the production of milligram amounts of c-di-GMP within 1 day and paves the way for extensive biochemical and biophysical studies on c-di-GMP-mediated processes.
\end{abstract}

Keywords c-di-GMP - Diguanylate cyclase $\cdot$ GGDEF domain $\cdot$ Enzymatic synthesis $\cdot$ E. coli

\section{Introduction}

Bis-( $\left.3^{\prime}-5^{\prime}\right)$-cyclic di-GMP (c-di-GMP) is an important bacterial second messenger involved in the regulation of a number of complex physiological processes. C-di-GMP was first identified 20 years ago as an activating factor of cellulose synthase in Acetobacter xylinum [1], but in the mean time, it has been shown to play a central role, among others, in the transition between a motile, single-cell state to a sessile, surface-attached state found in biofilms [2-5]. Its relevance for the virulence of pathogenic bacteria is well established [2-6].

C-di-GMP is synthesised by the condensation of two GTP molecules. This reaction is catalysed by the GGDEF domain of diguanylate cyclases (DGCs) [7-10], whereas EAL [7, 11-13] and HD-GYP [14] domains hydrolyse the compound to yield the linear pGpG

F. Zähringer $\cdot$ C. Massa $\cdot$ T. Schirmer $(\bowtie)$

Core Program Structural Biology and Biophysics, Biozentrum, University of Basel,

Klingelbergstrasse 70, CH-4056 Basel, Switzerland

e-mail: tilman.schirmer@unibas.ch 
dinucleotide. All these catalytic domains are typically found in combinations with other, mostly sensory or regulatory, domains and control the cellular level of c-di-GMP.

Much about the mechanisms of c-di-GMP signalling in bacteria has still to be discovered, in particular since only a few targets of c-di-GMP have been identified. Characterised c-di-GMP receptors comprise PilZ domains [15] and degenerated GGDEF and EAL domains [16, 17]. Furthermore, the GEMM riboswitches have recently been discovered to regulate gene expression via c-di-GMP binding [18-20].

Recent studies have demonstrated that exogenous c-di-GMP treatment inhibits adhesive Staphylococcus aureus cell-to-cell interactions and biofilm formation [21] and that it is effective also in a mouse model [22]. These findings make c-di-GMP an interesting candidate as a potential antimicrobial agent. It was demonstrated that c-di-GMP stimulates the immune system to prevent bacterial infections and is therefore evaluated as a potential vaccine adjuvant candidate [23-25]. It was also reported that c-di-GMP inhibits cancer cell proliferation in vitro and could therefore be used as a therapeutic agent [26].

In order to carry out extensive studies on c-di-GMP-mediated processes, sufficient supply of this compound is crucial. The reported chemical syntheses of c-di-GMP are multistep reactions, which make them time-consuming, expensive and inefficient [27-33]. Most of them make use of phosphotriester, phosphoamidite or H-phosphonate chemistry based on air- or water-sensitive reagents and involve several chromatographic purification steps.

In contrast, DGC-catalysed synthesis of c-di-GMP from GTP appears straightforward, as has been reported for PleD, VCA0956 and WspR [12, 13, 34-37]. However, all these DGCs show potent allosteric product inhibition with $K_{i}$ values in the low micromolar range. Furthermore, the purified proteins are rather unstable in solution. Recently, a DGC from a thermophilic organism (Thermotoga maritima) has been employed for the enzymatic synthesis of c-di-GMP [38]. A fragment comprising only its DGC domain with the allosteric inhibition site (I-site) mutated to impede product inhibition has been exploited. Large amounts (about $20 \mathrm{mg}$ ) of c-di-GMP were obtained employing $1 \mathrm{mg}$ of this modified version of the enzyme and by replenishing repeatedly the reaction mixture with the substrate GTP.

In this paper, we report a large-scale production procedure of c-di-GMP employing, as an alternative enzyme, YdeH from Escherichia coli. This enzyme consists of an N-terminal domain of unknown fold and a C-terminal GGDEF domain. We have shown previously [39] that $\mathrm{YdeH}$, in contrast to most of the other DGCs, is a constitutively active dimer and exhibits a turnover of $1.6 \mathrm{~min}^{-1}$ and a $K_{\mathrm{m}}$ of $17 \mu \mathrm{M}$. The YdeH catalysed reaction shows non-competitive product inhibition only at comparatively large c-di-GMP concentration $\left(K_{i}=44 \mu \mathrm{M}\right)$, in contrast to the aforementioned DGCs. Furthermore, even at very high c-di-GMP concentration $(>1 \mathrm{mM})$, YdeH shows residual activity (15\%) [39]. We show that with this enzyme, complete conversion of GTP to c-di-GMP can be achieved within hours, yielding milligram amount of product.

\section{Material and Methods}

\section{Expression and Purification of $\mathrm{YdeH}$}

C-terminally His6-tagged YdeH was expressed in the pET28b plasmid in the E. coli Rosetta strain. The transformed cells were cultivated at $37{ }^{\circ} \mathrm{C}$ in Luria-Bertani medium supplemented with ampicillin $(100 \mu \mathrm{g} / \mathrm{mL})$ and chloramphenicol $(34 \mu \mathrm{g} / \mathrm{mL})$ until the $\mathrm{OD}_{600}$ had reached 0.7 . Gene expression was induced by adding $1 \mathrm{mM}$ isopropyl- $\beta$-D-thiogalactopyranoside 
(IPTG). After $4 \mathrm{~h}$ of incubation, the cells were harvested by centrifugation $(6,800 \times g, 10 \mathrm{~min}$, $4{ }^{\circ} \mathrm{C}$ ) and the pellets were frozen at $-20{ }^{\circ} \mathrm{C}$.

Prior to lysis, the frozen cell pellets were thawed and resuspended in Ni-A buffer (50 mM NaH $\mathrm{PO}_{4}, \mathrm{pH} 7.5,200 \mathrm{mM} \mathrm{NaCl}, 10 \mathrm{mM}$ imidazole, $50 \mathrm{mM}$ L-glutamic acid, $50 \mathrm{mM}$ L-arginine). After the addition of DNAse $(2.5 \mu \mathrm{g} / \mathrm{mL}$; Sigma) and EDTA-free protease-inhibitor cocktail (Roche, 1 tablet $/ 50 \mathrm{~mL}$ buffer), the cells were disrupted with a French press (Thermo Spectronic) at 15,000 psi. The lysate was cleared by centrifugation $\left(28,000 \times \mathrm{g}, 45 \mathrm{~min}, 4{ }^{\circ} \mathrm{C}\right)$, and the supernatant was filtered $(0.22 \mu \mathrm{m})$ and loaded onto a $5-\mathrm{mL}$ HisTrap column (GE Healthcare). After washing the column with Ni-A buffer (10 column volumes $(\mathrm{CV})$ ), the protein was eluted with a linear gradient of imidazole from 10 to $500 \mathrm{mM}$ in $10 \mathrm{CV}$. The pooled fractions were concentrated to $1 \mathrm{~mL}$ and further purified by size-exclusion chromatography using a Superdex 75 16/60 column (GE Healthcare) and SEC buffer (20 mM Tris-HCl, pH 7.6, $150 \mathrm{mM} \mathrm{NaCl}, 50 \mathrm{mM}$ L-glutamic acid, $50 \mathrm{mM}$ L-arginine). The chromatographic runs were carried out with an Äkta Purifier FPLC unit and monitored with Unicorn software. The purification process was monitored by SDS-PAGE followed by Coomassie blue R-250 staining. Protein concentration was determined by measuring the absorbance at $280 \mathrm{~nm}\left(\varepsilon_{280}=39,880 \mathrm{M}^{-1} \mathrm{~cm}^{-1}\right)$.

\section{Enzyme Assay}

The initial rate of c-di-GMP synthesis was measured by following the substrate consumption using ion exchange chromatography. The reaction mixture $(100 \mu \mathrm{L})$ contained $2 \mu \mathrm{M}$ purified YdeH, $100 \mu \mathrm{M}$ GTP (Sigma), and $5 \mathrm{mM} \mathrm{MgCl}_{2}$. The effect of $\mathrm{pH}$ on the activity of YdeH was tested in $50 \mathrm{mM}$ MES, pH 6.5-7.5, $50 \mathrm{mM}$ Tris- $\mathrm{HCl}, \mathrm{pH}$ 7.5-8.5, and $50 \mathrm{mM}$ CHES, $\mathrm{pH} 9.0-10.0$. Also, the effect of different salts $(\mathrm{LiCl}, \mathrm{NaCl}$ and $\mathrm{KCl})$ and $\mathrm{NaCl}$ concentrations $(25 \mathrm{mM}-1.5 \mathrm{M})$ was investigated. The reaction was stopped by heating of the sample for $2 \mathrm{~min}$ at $99^{\circ} \mathrm{C}$. Subsequently, $100 \mu \mathrm{L}$ of the reaction mixture was diluted in $900 \mu \mathrm{L} 5 \mathrm{mM} \mathrm{NH} \mathrm{HCO}_{3}, \mathrm{pH} 8.0$, filtered $(0.22 \mu \mathrm{m})$ and loaded on an ionexchange column (ResourceQ $1 \mathrm{~mL}$, GE Healthcare). The nucleotides were separated with a gradient from 0.005 to $1 \mathrm{M} \mathrm{NH}_{4} \mathrm{HCO}_{3}, \mathrm{pH} 8.0$, in $14 \mathrm{CV}$. The amount of substrate and reaction product was determined by integration of the UV absorption $(253 \mathrm{~nm})$ peaks. The procedure was calibrated with GTP (Sigma) and c-di-GMP (Biolog, Bremen) standards of known concentrations.

\section{Enzymatic Production of c-di-GMP}

For the synthesis of c-di-GMP, $2 \mu \mathrm{M}$ YdeH was incubated with $500 \mu \mathrm{M}$ GTP in $50 \mathrm{mM}$ Tris- $\mathrm{HCl}, \mathrm{pH} 7.5,50 \mathrm{mM} \mathrm{NaCl}$ and $5 \mathrm{mM} \mathrm{MgCl}_{2}$ for $5 \mathrm{~h}$. The progress of the reaction was followed by withdrawing samples $(100 \mu \mathrm{L})$ that were inactivated by heat denaturation. Subsequently, the enzyme was removed by filtering and the sample content analysed by ion-exchange chromatography. After completion of the reaction, the mixture was heated for $5 \mathrm{~min}$ at $99^{\circ} \mathrm{C}$, centrifuged $(4,500 \times \mathrm{g}, 10 \mathrm{~min}$, room temperature $)$ and filtered $(0.22 \mu \mathrm{m})$. For purification of the reaction product, $\mathrm{Et}_{3} \mathrm{NHCO}_{3}$ to a final concentration of $5 \mathrm{mM}$ was added to the reaction mixture and loaded on a reversed-phase chromatography column (1.7 $\mathrm{mL}$ per $1 \mathrm{~mL}$ resin Resource RPC, GE Healthcare). After washing the column with $5 \mathrm{mM} \mathrm{Et}_{3} \mathrm{NHCO}_{3}(10 \mathrm{CV})$, c-di-GMP was eluted with a linear gradient of ethanol from $0 \%$ to $50 \%$ in $10 \mathrm{CV}$. The reversed-phase chromatography was carried out with an Äkta Purifier FPLC unit and monitored with Unicorn software. The c-di-GMP-containing fractions were pooled and lyophilised. The powder was dissolved in water and the concentration was 
determined by measuring the optical density at $253 \mathrm{~nm}$ of an aliquot diluted to $10 \mu \mathrm{M}$ (assuming an $\varepsilon_{253}$ of $23,700 \mathrm{M}^{-1} \mathrm{~cm}^{-1}$ as reported in [29]).

Analytical Methods

Purified c-di-GMP was analysed by high-performance liquid chromatography coupled to mass spectrometry. The samples were diluted 50-fold with $10 \mathrm{mM} \mathrm{NH}_{4} \mathrm{OAc}, \mathrm{pH} 6.4$, and injected into a Supercosil ${ }^{\mathrm{TM}}$ LC-18-T column (Supelco). The run was performed at a flow rate of $0.7 \mathrm{~mL} / \mathrm{min}$ using a linear gradient $(0-50 \%)$ of acetonitrile. The electron spray ionisation mass spectrometry with a time-of-flight analyser was performed using a microTOF Focus system (Bruker Daltronics). The capillary voltage was $4500 \mathrm{~V}$, and the end-plate offset was $500 \mathrm{~V}$ (negative mode); the dry temperature was $200{ }^{\circ} \mathrm{C}$, the dry gas flow was $9 \mathrm{~L} / \mathrm{min}$, and the nebulizer pressure was 2 bar.

To test the purity of the obtained c-di-GMP, a sample was analysed by ${ }^{1} \mathrm{H}$-nuclear magnetic resonance (NMR) spectroscopy (Bruker Advance 600) in $10 \mathrm{mM}$ Tris- $\mathrm{HCl}$ (D11), pH 7.4 (Cambridge Isotope Laboratories, Inc.), $150 \mathrm{mM} \mathrm{NaCl}, 50 \mu \mathrm{M}$ EDTA at 298 K. For comparison, spectra of synthetic c-di-GMP (Biolog) and GTP (Sigma) were acquired.

\section{Results and Discussion}

\section{Expression and Purification of $\mathrm{YdeH}$}

$Y d e H$ expression was tested in different $E$. coli strains and at different temperatures. In all conditions, most of the expressed $\mathrm{YdeH}$ was found in inclusion bodies. The highest amount of soluble protein was achieved in the Rosetta strain at $37^{\circ} \mathrm{C}, 3-4 \mathrm{~h}$ after IPTG induction. YdeH was purified to homogeneity using a two-step purification procedure, consisting of Ni-affinity and size-exclusion chromatography. To achieve a protein concentration above $0.8 \mathrm{mg} / \mathrm{mL}$, the addition of arginine and glutamic acid to the buffers was essential to avoid protein aggregation [40]. The obtained protein was pure and no degradation fragments were present, as demonstrated by the occurrence of a single band on SDS-PAGE (Fig. 1). The final yield was about $5 \mathrm{mgL}^{-1}$ culture.

Optimisation and Characterisation of the Enzymatic Reaction

Substrate and product amounts were analysed by ion-exchange chromatography. A peak at a somewhat smaller elution volume than that of the final product was observed in the

Fig. 1 SDS-PAGE of the purification of $\mathrm{YdeH}$. A $12 \%$ $(w / v)$ gel loaded with: lane 1 molecular weight marker, lane 2 cell extract, lane 3 soluble fraction of the cell extract, lane 4 purified $\mathrm{YdeH}$ after Ni column, lane 5 purified $\mathrm{YdeH}$ after size-exclusion chromatography

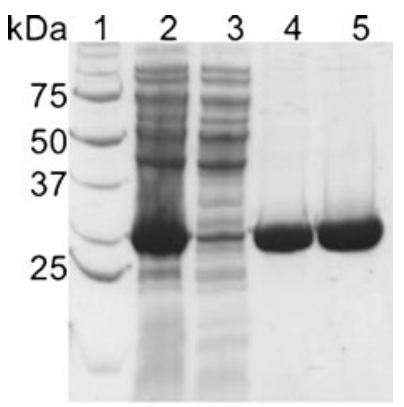


Fig. 2 Time course of the synthesis of c-di-GMP. The samples were analysed using ion-exchange chromatography. After $5 \mathrm{~h}$, the reaction is virtually complete. The leftmost small and constant peak corresponds to a GDP contamination in the commercial GTP batch

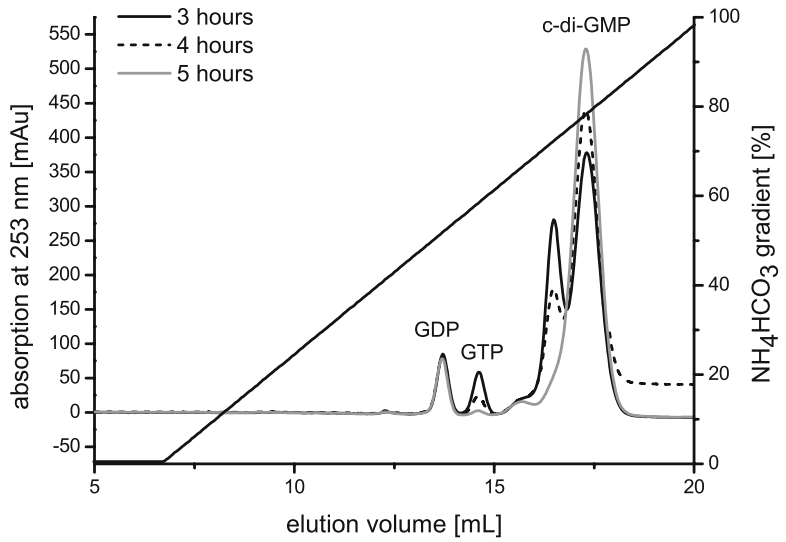

chromatograms taken at early to intermediate time points, but was absent after completion of the reaction (Fig. 2). As demonstrated by MS, this transient peak did not correspond to a reaction intermediate, but had the mass of c-di-GMP. Probably, the two peaks correspond to different oligomeric states of c-di-GMP.

The optimum $\mathrm{pH}$ for the synthesis of c-di-GMP was found to be at $\mathrm{pH} 7.5$ in Tris buffer (Fig. 3). Variation of the salt $(\mathrm{LiCl}, \mathrm{NaCl}, \mathrm{KCl})$ had only a marginal effect. The enzymatic activity was virtually the same in $50 \mathrm{mM} \mathrm{NaCl}$ and $50 \mathrm{mM} \mathrm{KCl}$, but was found to be reduced by about $20 \%$ in $50 \mathrm{mM} \mathrm{LiCl}$. Similarly, variation of the $\mathrm{NaCl}$ concentration (25 mM-1.5 M) had no significant effect on activity. Therefore, we chose a comparatively low $\mathrm{NaCl}$ concentration $(50 \mathrm{mM})$ to be compatible with the requirements for the subsequent c-di-GMP purification procedure.

Enzymatic c-di-GMP Production and Purification

For the large-scale synthesis of c-di-GMP, the molar ratio of enzyme to substrate has been optimised in order to obtain complete conversion within a convenient period. Using $2 \mu \mathrm{M}$ YdeH and $500 \mu \mathrm{M}$ GTP, virtually complete substrate conversion was achieved within $5 \mathrm{~h}$,

Fig. $3 \mathrm{pH}$ dependence of the enzymatic activity of $\mathrm{YdeH}$. The optimum reaction velocity for the synthesis of c-di-GMP was found in $50 \mathrm{mM}$ Tris- $\mathrm{HCl}$, $\mathrm{pH} 7.5$

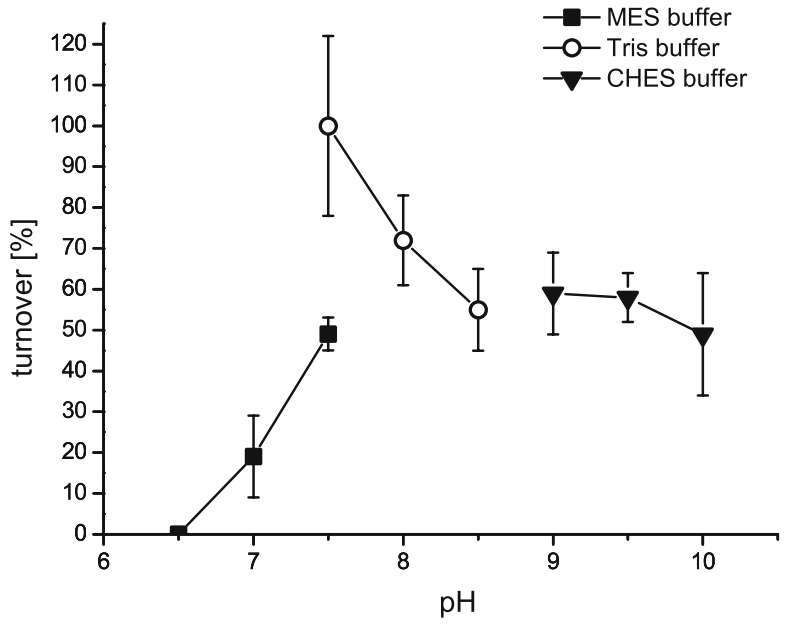




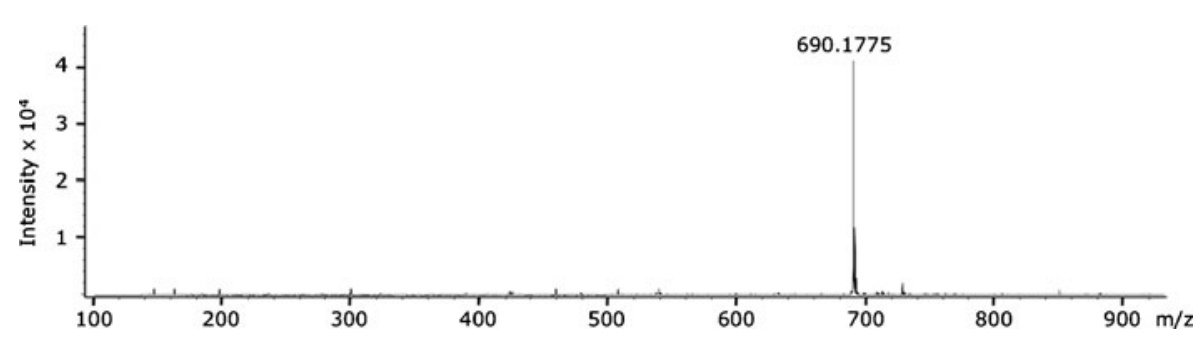

Fig. 4 Convoluted mass spectrum of enzymatically produced c-di-GMP. The measured mass of $690.1775 \mathrm{~g} /$ $\mathrm{mol}$ is in agreement with the calculated mass of $690.0870 \mathrm{~g} / \mathrm{mol}$

as shown in Fig. 2. Initially, the reaction volume was $0.1 \mathrm{~mL}$, but was eventually upscaled to $0.5 \mathrm{~L}$ without any detrimental effect. C-di-GMP was purified on a reversed-phase column with ethanol as eluent. The Resource RPC resin was chosen because of its stability at basic $\mathrm{pH}$ values. The mobile phase was adjusted so as to prevent binding of the mono-nucleotides GDP and GTP. Ethanol has the advantage of being non-toxic compared to other typically used eluents such as acetonitrile or methanol. The procedure yielded $75 \mathrm{mg}$ of pure c-di-GMP employing $30 \mathrm{mg}$ of YdeH. Considering the ease of YdeH purification, further optimisation of the procedure by recycling of the enzyme as in [38] was not considered.

Product Analysis

LC/MS clearly identified the reaction product as c-di-GMP (Fig. 4). No impurities were detected in the chromatogram. The observed mass of c-di-GMP was in perfect agreement with the calculated mass. In addition, the ${ }^{1} \mathrm{H}-\mathrm{NMR}$ spectra of purified c-di-GMP completely coincided with the spectrum of chemically synthesised c-di-GMP and showed no traces of the substrate GTP (Fig. 5).

\section{Conclusions}

Here, we have described an alternative way for the enzymatic synthesis of the bacterial second messenger c-di-GMP. We use the DGC YdeH from E. coli, which is an ideal

Fig. 5 Part of the ${ }^{1} \mathrm{H}-\mathrm{NMR}$ spectrum $(600 \mathrm{MHz})$ showing the chemical shift of $\mathrm{H} 8$ of enzymatically produced c-di-GMP (top, $\delta=8.04$ ) and of GTP (bottom, $\delta=8.13$ ) as reference. Comparison demonstrates that the c-di-GMP sample was free of GTP. Chemical shifts are relative to $\mathrm{H}_{2} \mathrm{O}(\delta=4.77$, 298 K)
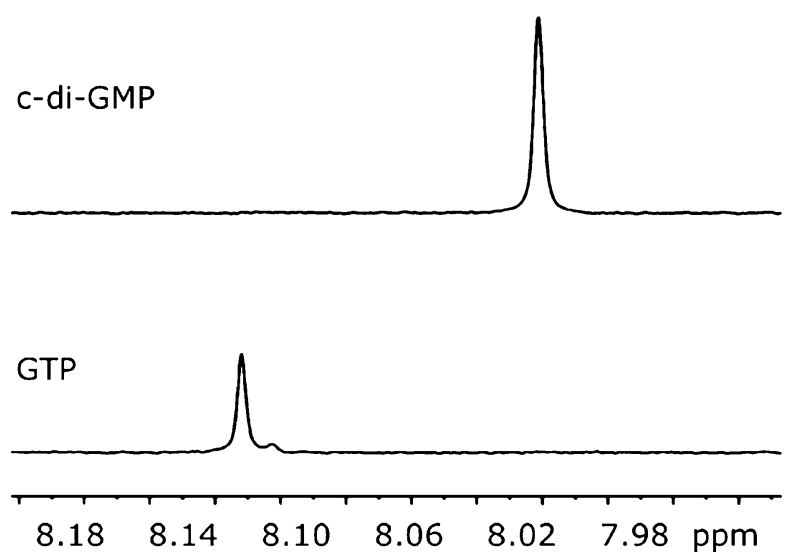
candidate because it is constitutively active in vitro and exhibits only weak product inhibition; thus, c-di-GMP concentrations of up to $0.25 \mathrm{mM}$ can be achieved. Our system allows producing $2.5 \mathrm{mg}$ of pure c-di-GMP per milligram of $\mathrm{YdeH}$ with standard biochemical lab equipment.

Enzymatic c-di-GMP production outperforms chemical synthesis with respect to time, costs and number of chromatographic purification steps [27-33]. Moreover, most of the chemical syntheses are based on air- and water-sensitive reagents. The enzymatic procedures described in the literature [12, 13, 34-37], however, employ rather unstable DGCs that, in addition, show strong product inhibition and therefore do not yield complete substrate conversion. The recently described procedure using a DGC from a thermophilic organism (T. maritima) [38] solved the solubility issue and provides the best specific yield so far (20 $\mathrm{mg} / \mathrm{mg}$ enzyme), but had to be mutated in the allosteric inhibition site (I-site) to abolish product inhibition.

Acknowledgement We thank Dr. Alexander Böhm, Biozentrum Basel, for the gift of the plasmid, Dr. Martin Allan, Biozentrum Basel, for help with ${ }^{1} \mathrm{H}-\mathrm{NMR}$ spectroscopy and Dietrich Samoray for suggesting to test the effect of osmolytes on YdeH solubility. The work was supported by Swiss National Science Foundation grant 3100A0-105587.

\section{References}

1. Ross, P., Weinhouse, H., Aloni, Y., Michaeli, D., Weinberger-Ohana, P., Mayer, R., et al. (1987). Regulation of cellulose synthesis in Acetobacter xylinum by cyclic diguanylic acid. Nature, 325, 279-381.

2. Jenal, U., \& Malone, J. (2006). Mechanisms of cyclic-di-GMP signaling in bacteria. Annual Review of Genetics, 40, 385-407.

3. Tamayo, R., Pratt, J. T., \& Camilli, A. (2007). Roles of cyclic diguanylate in the regulation of bacterial pathogenesis. Annual Review of Microbiology, 61, 131-148.

4. Schirmer, T., \& Jenal, U. (2009). Structural and mechanistic determinants of c-di-GMP signaling. Nature Reviews Microbiology, 7, 724-735.

5. Pesavento, C., \& Hengge, R. (2009). Bacterial nucleotide-based second messengers. Current Opinion in Microbiology, 12, 170-176.

6. Tamayo, R., Schild, S., Pratt, J. T., \& Camilli, A. (2008). Role of cyclic di-GMP during el tor biotype Vibrio cholerae infection: Characterization of the in vivo-induced cyclic di-GMP phosphodiesterase CdpA. Infection and Immunity, 76, 1617-1627.

7. Tal, R., Wong, H. C., Calhoon, R., Gelfand, D., Fear, A. L., Volman, G., et al. (1998). Three cdg operons control cellular turnover of cyclic di-GMP in Acetobacter xylinum: Genetic organization and occurrence of conserved domains in isoenzymes. Journal of Bacteriology, 80, 4416-4425.

8. Chan, C., Paul, R., Samoray, D., Amiot, N. C., Giese, B., Jenal, U., et al. (2004). Structural basis of activity and allosteric control of diguanylate cyclase. Proceedings of the National Academy of Sciences of the United States of America, 101, 17084-17089.

9. Paul, R., Weiser, S., Amiot, N. C., Chan, C., Schirmer, T., Giese, B., et al. (2004). Cell cycle-dependent dynamic localization of a bacterial response regulator with a novel di-guanylate cyclase output domain. Genes \& Development, 18, 715-727.

10. Ryjenkov, D. A., Tarutina, M., Moskvin, O. V., \& Gomelsky, M. (2005). Cyclic diguanylate is a ubiquitous signaling molecule in bacteria: Insights into biochemistry of the GGDEF protein domain. Journal of Bacteriology, 187, 1792-1798.

11. Schmidt, A. J., Ryjenkov, D. A., \& Gomelsky, M. (2005). The ubiquitous protein domain EAL is a cyclic diguanylate-specific phosphodiesterase: Enzymatically active and inactive EAL domains. Journal of Bacteriology, 187, 4774-4781.

12. Christen, M., Christen, B., Folcher, M., Schauerte, A., \& Jenal, U. (2005). Identification and characterization of a cyclic di-GMP-specific phosphodiesterase and its allosteric control by GTP. The Journal of Biological Chemistry, 280, 30829-30837. 
13. Rao, F., Yang, Y., Qi, Y., \& Liang, Z. X. (2008). Catalytic mechanism of cyclic di-GMP-specific phosphodiesterase: A study of the EAL domain-containing RocR from Pseudomonas aeruginosa. Journal of Bacteriology, 190, 3622-3631.

14. Galperin, M. Y. (2006). Structural classification of bacterial response regulators: Diversity of output domains and domain combinations. Journal of Bacteriology, 188, 4169-4182.

15. Amikam, D., \& Galperin, M. Y. (2006). PilZ domain is part of the bacterial c-di-GMP binding protein. Bioinformatics, 22, 3-6.

16. Duerig, A., Abel, S., Folcher, M., Nicollier, M., Schwede, T., Amiot, N., et al. (2009). Second messenger-mediated spatiotemporal control of protein degradation regulates bacterial cell cycle progression. Genes \& Development, 23, 93-104.

17. Tschowri, N., Busse, S., \& Hengge, R. (2009). The BLUF-EAL protein YcgF acts as a direct anti-repressor in a blue-light response of Escherichia coli. Genes \& Development, 15, 522-534.

18. Sudarsan, N., Lee, E. R., Weinberg, Z., Moy, R. H., Kim, J. N., Link, K. H., et al. (2008). Riboswitches in eubacteria sense the second messenger cyclic di-GMP. Science, 321, 411-413.

19. Smith, K. D., Lipchock, S. V., Ames, T. D., Wang, J., Breaker, R. R., \& Strobel, S. A. (2009). Structural basis of ligand binding by a c-di-GMP riboswitch. Nature Structural \& Molecular Biology, 16, 1218-1223.

20. Kulshina, N., Baird, N. J., \& Ferré-D'Amaré, A. R. (2009). Recognition of the bacterial second messenger cyclic diguanylate by its cognate riboswitch. Nature Structural \& Molecular Biology, 16, $1212-1217$.

21. Karaolis, D. K., Rashid, M. H., Chythanya, R., Luo, W., Hyodo, M., \& Hayakawa, Y. (2005). C-di-GMP (3'-5'-cyclic diguanylic acid) inhibits Staphylococcus aureus cell-cell interactions and biofilm formation. Antimicrobial Agents and Chemotherapy, 49, 1029-1038.

22. Brouillette, E., Hyodo, M., Hayakawa, Y., Karaolis, D. K., \& Malouin, F. (2005). 3'-5'-Cyclic diguanylic acid reduces the virulence of biofilm-forming Staphylococcus aureus strains in a mouse model of mastitis infection. Antimicrobial Agents and Chemotherapy, 49, 3109-3113.

23. Karaolis, D. K., Means, T. K., Yang, D., Takahashi, M., Yoshimura, T., Muraille, E., et al. (2007). Bacterial c-di-GMP is an immunostimulatory molecule. Journal of Immunology, 178, 2171-2181.

24. Karaolis, D. K., Newstead, M. W., Zeng, X., Hyodo, M., Hayakawa, Y., Bhan, U., et al. (2007). Cyclic di-GMP stimulates protective innate immunity in bacterial pneumonia. Infection and Immunity, 75 , 4942-5450.

25. Ogunniyi, A. D., Paton, J. C., Kirby, A. C., McCullers, J. A., Cook, J., Hyodo, M., et al. (2008). C-di-GMP is an effective immunomodulator and vaccine adjuvant against pneumococcal infection. Vaccine, 26, 46764685 .

26. Karaolis, D. K., Cheng, K., Lipsky, M., Elnabawi, A., Catalano, J., Hyodo, M., et al. (2005). 3',5'-Cyclic diguanylic acid (c-di-GMP) inhibits basal and growth factor-stimulated human colon cancer cell proliferation. Biochemical and Biophysical Research Communications, 329, 40-45.

27. Ross, P., Mayer, R., Weinhouse, H., Amikam, D., Huggirat, Y., Benziman, M., et al. (1990). The cyclic diguanylic acid regulatory system of cellulose synthesis in Acetobacter xylinum. Chemical synthesis and biological activity of cyclic nucleotide dimer, trimer, and phosphothioate derivatives. The Journal of Biological Chemistry, 265, 18933-18943.

28. Kawai, R., Nagata, R., Hirata, A., \& Hayakawa, Y. (2003). A new synthetic approach to cyclic bis $\left(3^{\prime} \rightarrow 5^{\prime}\right)$ diguanylic acid. Nucleic Acids Symposium Series, 3, 103-104.

29. Hayakawa, Y., Nagata, R., Hirata, A., Hyodo, M., \& Kawai, R. (2003). A facile synthesis of cyclic bis $\left(3^{\prime} \rightarrow 5^{\prime}\right)$ diguanylic acid. Tetrahedron, 59, 6465-6471.

30. Zhang, Z., Gaffney, B. L., \& Jones, R. A. (2004). C-di-GMP displays a monovalent metal ion-dependent polymorphism. Journal of the American Chemical Society, 26, 16700-16701.

31. Amiot, N., Heintz, K., \& Giese, B. (2006). New approach for the synthesis of c-di-GMP and its analogues. Synthesis, 24, 4230-4236.

32. Yan, H., \& Aguilar, A. L. (2007). Synthesis of $3^{\prime}, 5^{\prime}$-cyclic diguanylic acid (cdiGMP) using 1-(4chlorophenyl)-4-ethoxypiperidin-4-yl as a protecting group for 2'-hydroxy functions of ribonucleosides. Nucleosides, Nucleotides \& Nucleic Acids, 26, 189-204.

33. Kiburu, I., Shurer, A., Yan, L., \& Sintim, H. O. (2008). A simple solid-phase synthesis of the ubiquitous bacterial signaling molecule, c-di-GMP and analogues. Molecular Biosystems, 4, 518-520.

34. Tamayo, R., Tischler, A. D., \& Camilli, A. (2005). The EAL domain protein VieA is a cyclic diguanylate phosphodiesterase. The Journal of Biological Chemistry, 280, 33324-33330.

35. Kazmierczak, B. I., Lebron, M. B., \& Murray, T. S. (2006). Analysis of FimX, a phosphodiesterase that governs twitching motility in Pseudomonas aeruginosa. Molecular Microbiology, 60, 1026-1043.

36. Merighi, M., Lee, V. T., Hyodo, M., Hayakawa, Y., \& Lory, S. (2007). The second messenger bis-(3'-5')cyclic-GMP and its PilZ domain-containing receptor Alg44 are required for alginate biosynthesis in Pseudomonas aeruginosa. Molecular Microbiology, 65, 876-895. 
37. Hickman, J. W., \& Harwood, C. S. (2008). Identification of FleQ from Pseudomonas aeruginosa as a c-di-GMP-responsive transcription factor. Molecular Microbiology, 69, 376-389.

38. Rao, F., Pasunooti, S., Ng, Y., Zhuo, W., Lim, L., Liu, A. W., et al. (2009). Enzymatic synthesis of c-di-GMP using a thermophilic diguanylate cyclase. Analytical Biochemistry, 389, 138-142.

39. Böhm, A., Steiner, S., Zähringer, F., Casanova, A., Hamburger, F., Ritz, D., et al. (2009). Second messenger signalling governs Escherichia coli biofilm induction upon ribosomal stress. Molecular Microbiology, 72, 1500-1516.

40. Golovanov, A. P., Hautbergue, G. M., Wilson, S. A., \& Lian, L. Y. (2004). A simple method for improving protein solubility and long-term stability. Journal of the American Chemical Society, 126, 8933-8939. 